\title{
Super-selective Partial Splenic Embolization for Hereditary Spherocytosis in Children: A Single-Center retrospective Study
}

\author{
Qiang Xiong ${ }^{1}$, Ruijue Wang ${ }^{1}, \mathrm{Li}_{\mathrm{Xiao}}{ }^{1}, \mathrm{Xiaoke}_{\mathrm{Dai}}{ }^{1},{\text { Yingcun } \mathrm{Li}^{1} \text {, and Mingman Zhang }}^{1}$ \\ ${ }^{1}$ Chongqing Medical University Affiliated Children's Hospital
}

March 16, 2021

\begin{abstract}
Hereditary spherocytosis is the most common cause of hemolytic anemia due to defects in the blood cell membrane. Partial splenic embolization (PSE) might significantly improve the effects of hemolysis in HS patients while preserving partial splenic function. However, due to the poor controllability of the scope of interventional embolization, PSE in pediatric HS had not been widely studied and applied. The present study is the first to propose that optimized PSE procedure to treat pediatric HS patients, which named super-selective partial splenic embolization (SPSE). We investigated the feasibility and effectiveness of SPSE. This study was conducted by retrospectively reviewing clinical data from HS patients treated by surgical treatments who were diagnosed at children's hospital of Chongqing medical university from January 2015 to December 2019. Patients were divided into two groups according to treatment preference: SPSE (16 patients) group and total splenectomy (41 patients) group. The data were analyzed such as preoperative and postoperative leukocytes, hemoglobin, platelets, bilirubin, immunological indicators, and postoperative complications. The success rate of two groups were $100 \%$. The improvement of hemolysis in patients after SPSE was not different from that after total splenectomy, and the effect can be maintained for a long time. The average hemoglobin value increased significantly from $6.97 \mathrm{~g} / \mathrm{dL}$ before SPSE to $12.2 \mathrm{~g} / \mathrm{dL}$ after SPSE (P=0.000). SPSE was performed without any serious complications. SPSE is a safe and effective treatment for moderate or severe HS in children.
\end{abstract}

\section{Introduction}

Hereditary spherocytosis (HS) is the most common cause of hemolytic anemia due to defects in the blood cell membrane. Total splenectomy is the most effective treatment modality for moderate or severe HS, but young children are at risk of post-splenectomy infections. Partial splenic embolization (PSE) might significantly improve the effects of hemolysis in HS patients while preserving partial splenic function. However, due to the poor controllability of the scope of interventional embolization, PSE in pediatric HS had not been widely studied and applied. The present study is the first to propose that optimized PSE procedure to treat pediatric HS patients, which named super-selective partial splenic embolization (SPSE). We investigated the feasibility and effectiveness of SPSE.

\section{Methods}

This study was conducted by retrospectively reviewing clinical data from HS patients treated by surgical treatments who were diagnosed at children's hospital of Chongqing medical university from January 2015 to December 2019. Patients were divided into two groups according to treatment preference: SPSE (16 patients) group and total splenectomy (41 patients) group. The data were analyzed such as preoperative and postoperative leukocytes, hemoglobin, platelets, bilirubin, immunological indicators, and postoperative complications.

\section{Results}

The success rate of two groups were 100\%, and the mean degree of splenic embolism by SPSE was $82.4 \%$ 
(75\%-90\%), which close to the extent of expected embolism. The improvement of hemolysis in patients after SPSE was not different from that after total splenectomy, and the effect can be maintained for a long time. The average hemoglobin value increased significantly from $6.97 \mathrm{~g} / \mathrm{dL}$ before SPSE to $12.2 \mathrm{~g} / \mathrm{dL}$ after SPSE $(P<0.001)$. SPSE was performed without any serious complications. All children suffered mild to moderate postembolization syndrome including pain, fever and vomiting, which could easily be controlled with appropriate supportive therapy.

\section{Conclusions}

SPSE is a safe and effective treatment for moderate or severe HS in children. SPSE may have greater advantages by expanding the age range of the procedure, reducing surgical trauma, and reducing complications such as infection. However, a longer follow-up, a greater number of patients and to apply on other disease such as hypersplenism are mandatory to further assess the value of SPSE.

\section{Keywords:}

\section{Introduction}

Hereditary spherocytosis (HS) is a hemolytic anemia of varying severity caused by defects in the red blood cell membrane. Defects in the erythrocyte membrane and selective destruction of defective erythrocytes by the spleen are two key factors in the pathophysiological process of HS. The destruction of the red blood cells by the spleen can lead to proliferative enlargement of the spleen, which leads to increased red blood cell accumulation and/or increased hemolysis, thus creating a vicious cycle ${ }^{1}$.

The treatment of HS is aimed at minimizing the complications of chronic hemolysis and anemia. In addition to symptomatic treatment and blood transfusion, current guidelines recommend splenectomy if the patient has severe anemia complications or is transfusion dependent ${ }^{1-3}$. Since the spleen is a lymphoid organ, the overwhelming post-splenectomy infection (OPSI) is the most serious postoperative complication, especially in children. In addition to surgical risks, splenic resection is also prone to serious surgical complications such as venous thromboembolism (VTE), pulmonary arthritis, and pulmonary artery hypertension (PAH) after splenectomy ${ }^{4}$. Patients who have indications for splenectomy but were unwilling to undergo surgery, or who have contraindications to surgery, have been reported to be treated with PSE, which exhibited better outcomes and fewer surgical complications due to residual partial splenic immunity ${ }^{5,6}$. However, PSE had not been widely studied and applied in pediatric HS patients due to the poor controllability of the embolic range.

The aim of our study was to investigate another surgical procedure, which can achieve an accurate prediction of the extent of splenic embolism. We know that the splenic artery near the splenic hilum is usually divided into two branches at the upper and lower ends, each of which is further divided into 4-6 segmental intrapleural branches, with few traffic branches between these different segments. Thus, we first proposed SPSE to treat HS patients based on the anatomical characteristics of the splenic artery. The present study reports on the feasibility and efficacy of SPSE in a cohort of 16 children with HS.

\section{Patients and method}

\section{Study patients}

This study was conducted by retrospectively reviewing clinical data from a total of 58 children patients with HS who were diagnosed at the Children's Hospital of Chongqing Medical University from January 2015 to December 2019. Our study was approved by the ethics committee of the Children's Hospital of Chongqing Medical University (No: 2020-288), and informed consent was obtained to review patient medical records. We excluded one patient with splenectomy because follow-up data were lacking, leaving 57 patients for the study. All children presented with an enlarged spleen. All eligible patients were assessed according to the BCSH 2011 guidelines and European Hematology Association 2017 guidelines $^{2,3}$, while only patients with moderate, severe HS or impact daily life were operated on. Grouping was based on treatment intention 41 patients had undergone total splenectomy (median age 9, range 2-17 years) and 16 patients had undergone 
SPSE (median age 7 years, range 5-11 years). All patients or guardians were informed about the advantages and disadvantages of the procedure and received a written informed consent prior to surgery.

\section{SPSE surgery procedure}

We performed selective embolization of the splenic segmental artery based on the unique anatomy of the spleen and splenic artery by analyzing the distribution of the splenic segmental artery by means of splenic artery trunk angiography. At the same time, the degree of embolization was judged by the intraoperative flow velocity of the embolized artery. The degree of embolization was determined according to a slight slowing of the flow as $30 \%$ to $40 \%$, a marked slowing as $50 \%$ to $60 \%$, a peristaltic advance after briefly stopped as $70 \%$ to $80 \%$, a marked regurgitation as $90 \%$ and above. The extent of splenic embolism was predicted by the above two aspects. The specific surgical procedure is as follows:

First, splenic arteriogram procedure. The patients were placed in the supine position, a 4Fr introducer sheath (RS*A40G07SQ, TERUMO) was inserted through the right common femoral artery by using the Seldinger technique and a 4Fr catheter (RF*ZB54110, TERUMO) was passed through the main trunk of the splenic artery for imaging. Based on the visualization of the splenic vessels, adopting secondary artery in the lower and middle spleen as the target vessel, the arterial embolization, which was confirmed by contrast, was performed by a $2.7 \mathrm{Fr}$ microcatheter (MC-PE27131, TERUMO) to super-select the grade 2-3 arterial branches.

Second, configuration of embolic agents. Mixing polyvinyl alcohol granules (PVA-500, COOK)1g and lodixanol320 (GE Healthcare Ireland) $5 \mathrm{ml}$ thoroughly, extract $2 \mathrm{ml}$ of suspension and add $0.25 \mathrm{~g}$ of triple cephalosporin, then add the appropriate amount of contrast agent to make a total of $15 \mathrm{ml}$ of embolic agent.

Third, embolization procedure. The embolization area was mainly in the middle and lower spleen, preserving most of the upper spleen and the splenic hilum. After completion of embolization, the degree of embolization was determined again by imaging of the main trunk of the splenic artery. If the embolic scope was insufficient, additional embolization operations can be performed until the embolic scope reaches $70 \%-85 \%$. Sterile gauze and pressure were applied to the puncture site for hemostasis followed by bandaging and braking for 6-8 hours. The operation time only require 30 minutes maximum. The patency of splenic vein flow was detected by vascular ultrasound 3 days after surgery, and third-generation cephalosporin was applied by intravenous prophylactic in 48 hours after surgery.

\section{Data collection and follow-up}

We collected preoperative and postoperative examination data of patients who underwent total splenectomy and SPSE, with a minimum 6-months follow-up. The last follow-up was in September 2020. The patients underwent computed tomography (CT) scan using a 64-channel multidetector CT system (Lig_x0002_htspeed VCT, GE Medical systems, USA). Splenic volume were analyzed by Volume Rendering function with the philips intellispace portal software platform, version 6.0.1.20700.

\section{Statistical methods}

Statistical analyses were performed using the SPSS software version 22.0. Measurement data were expressed as mean \pm standard deviation $( \pm \mathrm{sd})$ and analyzed by Student's $t$-test. Enumeration data were expressed as rate (\%), and Chi-square test was adopted. Comparison of laboratory parameters after splenic artery embolization was carried out using the paired t-test, the Wilcoxon signed ranks test, the Mann-Whitney U test, or the chi-square test. Statistical significance was defined as $P<0.05$.

\section{Results}

\section{Comparison of general clinical data between total splenectomy and SPSE}

The surgical success rate was $100 \%$. No cases of serious postoperative complications or post-splenectomy infections were found in either group within one month after surgery during the follow-up period. There was no statistical difference in the preoperative condition of the two groups (TABLE 1), and hemolysis was 
significantly improved in both groups. The average hemoglobin value increased significantly from $7.4 \mathrm{~g} / \mathrm{dL}$ before total splenectomy to $11.7 \mathrm{~g} / \mathrm{dL}$ after total splenectomy $(P<0.001)$. The average hemoglobin value increased significantly from $6.97 \mathrm{~g} / \mathrm{dL}$ before SPSE to $12.2 \mathrm{~g} / \mathrm{dL}$ after SPSE $(P<0.001)$. Compared to total splenectomy, patients who had undergone PSAE exhibited higher platelet counts within 1 month postoperatively $(P ; 0.05)$.

\section{General condition and treatment outcome of patients undergoing SPSE}

The surgical results were in table 2. Before and after embolization, the arterial phase showed the branches and distribution of the splenic arteries, and the equilibrium phase showed the distribution of the parenchymal blood supply to the spleen (Fig. 1).

The success rate of the SPSE procedure was 100\%. 16 children underwent embolization of the middle and lower pole of the spleen, and the expected intraoperative embolization extent was $75 \%-90 \%$. Preembolization and post-embolization (7-9 days) CT examinations were completed in 11 patients. The preand post-embolization spleen volumes were calculated and the actual splenic embolic extent was $73.2 \%-89.1 \%$ (Fig. 2). The difference between the pre-assessed embolic extent and the actual postoperative embolic extent was not statistically significant. This result suggests that SPSE can accurately determine the extent of splenic embolization intraoperatively. For preoperatively larger spleens, such as larger than $550 \mathrm{~cm}^{3}$, there may be a small deviation of approximately $10 \%$ in the assessment results.

No serious complications had occurred in any patient treated with SPSE (TABLE 2). All children experienced mild to moderate postembolization syndrome. $87.5 \%$ (14/16) of children presented with mild abdominal pain with a median abdominal pain duration of 6 days (range 0-9 days), which could easily be controlled with appropriate supportive therapy. Postoperative fever and vomiting occurred in $25.0 \%(4 / 16)$ and $31.3 \%(5 / 16)$ of the children, both of which resolved rapidly on autonomy. No other serious complications such as splenic abscess, portal vein embolism, or pulmonary atelectasis occurred.

\section{Comparison of preoperative and postoperative clinical indicators in patients with SPSE}

The median follow-up time for this group of patients was 19 months (8-26 months), all children showed significant improvement in hemolysis, and no patient underwent transfusion for hemolysis during the followup period. Notably, there were no significant abnormalities in the common immune indicators before and after surgery, suggesting that the patient retained splenic immune function after surgery (TABLE 3 ).

\section{Discussion}

Total splenectomy is a proven and effective method for the treatment of patients with moderate to severe $\mathrm{HS}^{3}$. The spleen is the largest peripheral lymphoid organ in the body, it is an indispensable immune barrier when the body receives external viruses and bacteria. The absence of spleen might lead an infection outbreak. For pediatric patients, possible postoperative aggressive complications such as OPSI and surgical trauma have been a long-term problem for physicians and patients. In 1973, Maddison ${ }^{7}$ proposed splenic artery embolization as a minimally invasive procedure for the treatment of hypersplenism that solves the problem of severe trauma. Spigos ${ }^{8}$ et al. developed transcatheter partial splenic embolization (PSE) in adult HS to preserve the immune function of the spleen ${ }^{5,6}$. However, PSE has not been widely promoted in pediatric patients because of the poor controllability of the extent of PSE embolization.

In our study, all the patients had moderate or severe HS. The success rate of splenectomy and SPSE was 100\%. Postoperative hemolysis was significantly improved, no further transfusions were performed in our patients. Previous studies had shown that PSE was effective in reducing the degree of hemolysis while maintaining the phagocytic function of the splenic remnant, and the selective destruction of the spleen to defective red blood cells $^{5}$. In this study, based on the unique anatomy of the spleen and splenic artery, selective embolization of grade 2 to grade 3 arterial branches was performed in the spleen by intraoperative angiography. Measuring the embolic artery blood flow velocity and performing a comprehensive pre-evaluation of the extent of splenic embolization to achieving an accurate prediction of splenic embolic volume. The extent of spleen volume 
that examined by CT before and after embolization was $75 \%$ to $90 \%$, which was consistent with the preevaluation results. Therefore, we believe the degree of splenic embolization can be precisely controlled by SPSE to achieve a therapeutic effect of reducing hemolysis.

The white marrow of the spleen is an important component of the lymphoid tissue in the body (Approximately $25 \%$ ). It has germinal centers which dominated by early B lymphocytes and plasma cells. Therefore, total splenectomy can lead to a significant reduction in antibody production. When foreign antigens are appeared in the circulation, the spleen provides an essential place for the immune response ${ }^{9}$. In this study, SPSE can effectively reduce the volume of the spleen and maintain the immune function of the splenic white marrow to some extent. The mild elevation of the complement system compared to the preoperative period was considered an effect possibly relate to surgical embolization ${ }^{10,11}$. The complement system exerts innate immune by lysing or killing of bacteria, modulating phagocytosis or participates in antibody-mediated specific immune responses. Our study also showed that all splenic immune functions were maintained in the normal range after SPSE and no surgery-related infection of SPSE during the follow-up period. Thus, we demonstrated SPSE had a substantial improvement in maintaining normal immune function than total splenectomy. We are aware that the younger the patients with HS diagnosis, the severer the symptoms of hemolytic anemia may have. Considering the risk of post-splenectomy fulminant infection, many patients with total splenectomy was significantly older. We believed SPSE preserves normal immune function while treating HS, offers the possibility of surgery in earlier ages and reduces the complications for patients with HS.

All the post-embolization syndromes in this study are minor and controllable, it mainly manifested by abdominal pain, fever and vomiting. Most patients' symptoms resolve within 1 week after surgery. Previous studies have shown that common complications of total splenectomy include postoperative infection, OPSI, surgical bleeding, injury of adjacent organs and venous thromboembolism (VTE) ${ }^{2,12,13}$. The most serious complications of PSE are abdominal infection and spleen abscess formation, which is deadly for patients in severe cases ${ }^{14}$. The cause of spleen abscess formation includes excessive embolization which leads liquefied necrosis of a large amount of spleen tissue and secondary bacterial infection. There are two possible sources of bacteria, one of them is interventional contamination, the other is that, the enteric-derived bacteria which retained in the portal venous system reverse into the spleen since the slow speed of flow in the splenic vein after massive embolization of the splenic parenchyma ${ }^{15}$. However, there is no patient had a splenic abscess in this study. The reason to do this study is as following. Firstly, the number of patients is still small. Secondly, SPSE achieved the purpose that accurately embolization by the accurate selection of secondary arteries and narrowing the possibility of infection caused by stagnation of splenic vein blood flow which is the result of over embolization of splenic artery trunk. Thirdly, the minified dosage of embolic agent during treatment also indirectly diminishes the risk of contamination. The fact that there is not any expose of significant flow stagnation or reflux in the splenic artery angiogram after SPSE had been confirmed by the color ultrasonography of the blood flow of splenic vein 3 days after the procedure. In addition, triple cephalosporin was intravenously applied within 48 hours postoperatively. Since these prophylactic antiinfection actions were actively performed, the risk of splenic infection was minimized by avoiding possible intraoperative contamination and postoperative enterogenic infection to the greatest extent.

A few reports of previous study shown the growth of residual accessory spleen after total splenectomy ${ }^{16}$, or growth of residual spleen after partial splenectomy trigger recurrence of anemia which requires secondary surgery ${ }^{17,18}$. In a long-term follow-up study of partial splenectomy ${ }^{19}$, mild and moderate hemolysis may be a long term symptoms after partial splenectomy, and a small number of patients may be at risk of secondary gallstones and hemolytic anemia. In our observed SPSE patients with a median follow-up time of 19 months (8-26 months), there is no cases of hemolytic anemia which requires transfusion therapy, only a few children have mild hemolysis and all patients with SPSE recovered well after surgery. We believe the better results were mainly becauseonly small portion of the upper splenic (10\%-20\%) were preserved by the accurate control of embolization, moreover, the growth of the normal residual spleen was effectively limited by the blockage of diaphragm and embolized spleen. In fact, since we preserved the splenic artery trunk upfront, there is an opportunity to do another embolization as re-intervention if symptomatic recurrence of anemia appeared at 
later stage. The shortage of this study is the short follow-up period. Hence, long-term follow-up should be applied to clarify the dynamic changes of spleen volume and hemolysis after embolization.

In conclusion, SPSE is a feasible, safe and effective alternative to splenectomy for childhood hereditary spherocytosis. SPSE may have greater advantages on expanding the age range of surgery, reducing the severity of surgical trauma, and minimizing the possibility of infection. However, a longer follow-up period, a larger sample size, and applying other indications such as hypersplenism, are mandatory for the further assessment of SPSE.

\section{Acknowledgments}

The authors would like to thank all families who generously accept to participate in this study. The authors would like to thank the doctors and nurses of CHCMU for their facilitation of patients for the study. The authors also gratefully thanks to Xiao Li for his outstanding work in the language editing.

\section{Funding}

This study has not received external funding.

\section{Authors' contributions}

Qiang Xiong and Ming-man Zhang conceived the study and is the principal investigator. Qiang Xiong and Ming-man Zhang initially designed the study protocol. Ming-man Zhang coordinated the doctors and nurses. At the same time, Qiang Xiong, Ruijue Wang, Xiaoke Dai, Yingcun Li and Ming-man Zhang mainly provided clinical support and lead surgery. Li Xiao and Ruijue Wang contributed to subsequent amendments to the study protocol. Qiang Xiong and Ruijue Wang wrote the first draft of this manuscript. Ruijue Wang and Li Xiao revised this manuscript. All authors contributed to subsequent drafts and approved the final manuscript.

\section{Ethics approval and consent to participate}

Ethical approval for this study was provided by the Institutional Review Board of CHCMU and registered at No: $2020-288$.

\section{Competing interests}

No conflicting financial interests exist.

\section{References}

1. Perrotta S, Gallagher PG, Mohandas N. Hereditary spherocytosis.Lancet . 2008;372(9647):1411-1426. doi:10.1016/S0140-6736(08)61588-3

2. Iolascon A, Andolfo I, Barcellini W, et al. Recommendations regarding splenectomy in hereditary hemolytic anemias. Haematologica . 2017;102(8):1304-1313. doi:10.3324/haematol.2016.161166

3. Bolton-Maggs PHB, Langer JC, Iolascon A, Tittensor P, King MJ. Guidelines for the diagnosis and management of hereditary spherocytosis - 2011 update. Br J Haematol . 2012;156(1):37-49. doi:10.1111/j.13652141.2011.08921.x

4. Smedema JP, Louw VJ. Pulmonary arterial hypertension after splenectomy for hereditary spherocytosis. Cardiovasc J Afr . 2007;18(2):84-89.

5. Pratl B, Benesch M, Lackner H, et al. Partial splenic embolization in children with hereditary spherocytosis. Eur J Haematol . 2008;80(1):76-80. doi:10.1111/j.1600-0609.2007.00979.x

6. Kimura F, Ito H, Shimizu H, et al. Partial Splenic Embolization for the Treatment of Hereditary Spherocytosis. Am J Roentgenol . 2003;181(4):1021-1024. doi:10.2214/ajr.181.4.1811021

7. Maddison F E. Embolic Therapy of Hypersplenism.pdf. Invest Radiol . 1973;8(4):280-281. 
8. Spigos D, Jonasson O, Mozes M, Capek V. Partial splenic embolization in the treatment of hypersplenism. Am J Roentgenol . 1979;132(5):777-782. doi:10.2214/ajr.132.5.777

9. Tussiwand R, Bosco N, Ceredig R, Rolink AG. Tolerance checkpoints in B-cell development: Johnny B good. Eur J Immunol . 2009;39(9):2317-2324. doi:10.1002/eji.200939633

10. Walport MJ. Complement. First of two parts. $N$ Engl J Med . 2001;344(14):1058-1066. doi:10.1056/NEJM200104053441406

11. Walport MJ. Advances in immunology: Complement (second of two parts). $N$ Engl J Med . 2001;344(15):1140-114. doi:10.1056/NEJM200104123441506

12. Perkins LA, Jones SF, Bhargava RS. Dural venous thrombosis following splenectomy in a patient with hereditary spherocytosis. South Med J . 2009;102(5):542-545. doi:10.1097/SMJ.0b013e31819e90b5

13. Davidsen C, Larsen TH, Gerdts E, Lonnebakken MT. Giant right ventricular outflow tract thrombus in hereditary spherocytosis: A case report. Thromb J . 2016;14(1). doi:10.1186/s12959-016-0083-3

14. Cai M, Huang W, Lin C, et al. Partial splenic embolization for thrombocytopenia in liver cirrhosis: predictive factors for platelet increment and risk factors for major complications. Eur Radiol . 2016;26(2):370380. doi:10.1007/s00330-015-3839-4

15. Masada T, Tanaka T, Sakaguchi H, et al. Coils versus gelatin particles with or without intraarterial antibiotics for partial splenic embolization: A comparative evaluation. J Vasc Interv Radiol . 2014;25(6):852858. doi:10.1016/j.jvir.2013.12.563

16. Mackenzie FAF, Elliot DH, Eastcott HHG, Bakkhan P, Mollison PL. RELAPSE IN HEREDITARY SPHEROCYTOSIS WITH PROVEN SPLENUNCULUS.Lancet . 1962;279(7239):1102-1104. doi:10.1016/S0140-6736(62)92084-6

17. De Buys Roessingh AS, De Lagausie P, Rohrlich P, Berrebi D, Aigrain Y. Follow-up of partial splenectomy in children with hereditary spherocytosis. J Pediatr Surg . 2002;37(10):1459-1463. doi:10.1053/jpsu.2002.35412

18. Rice HE, Oldham KT, Hillery CA, Skinner MA, O'Hara SM, Ware RE. Clinical and Hematologic Benefits of Partial Splenectomy for Congenital Hemolytic Anemias in Children. Ann Surg . 2003;237(2):281-288. doi:10.1097/01.SLA.0000048453.61168.8F

19. Bader-Meunier B, Gauthier F, Archambaud F, et al. Long-term evaluation of the beneficial effect of subtotal splenectomy for management of hereditary spherocytosis. Blood . 2001;97(2):399-403. doi:10.1182/blood.V97.2.399

\section{Hosted file}

TABLE.pdf available at https://authorea.com/users/401967/articles/513900-super-selectivepartial-splenic-embolization-for-hereditary-spherocytosis-in-children-a-single-centerretrospective-study 

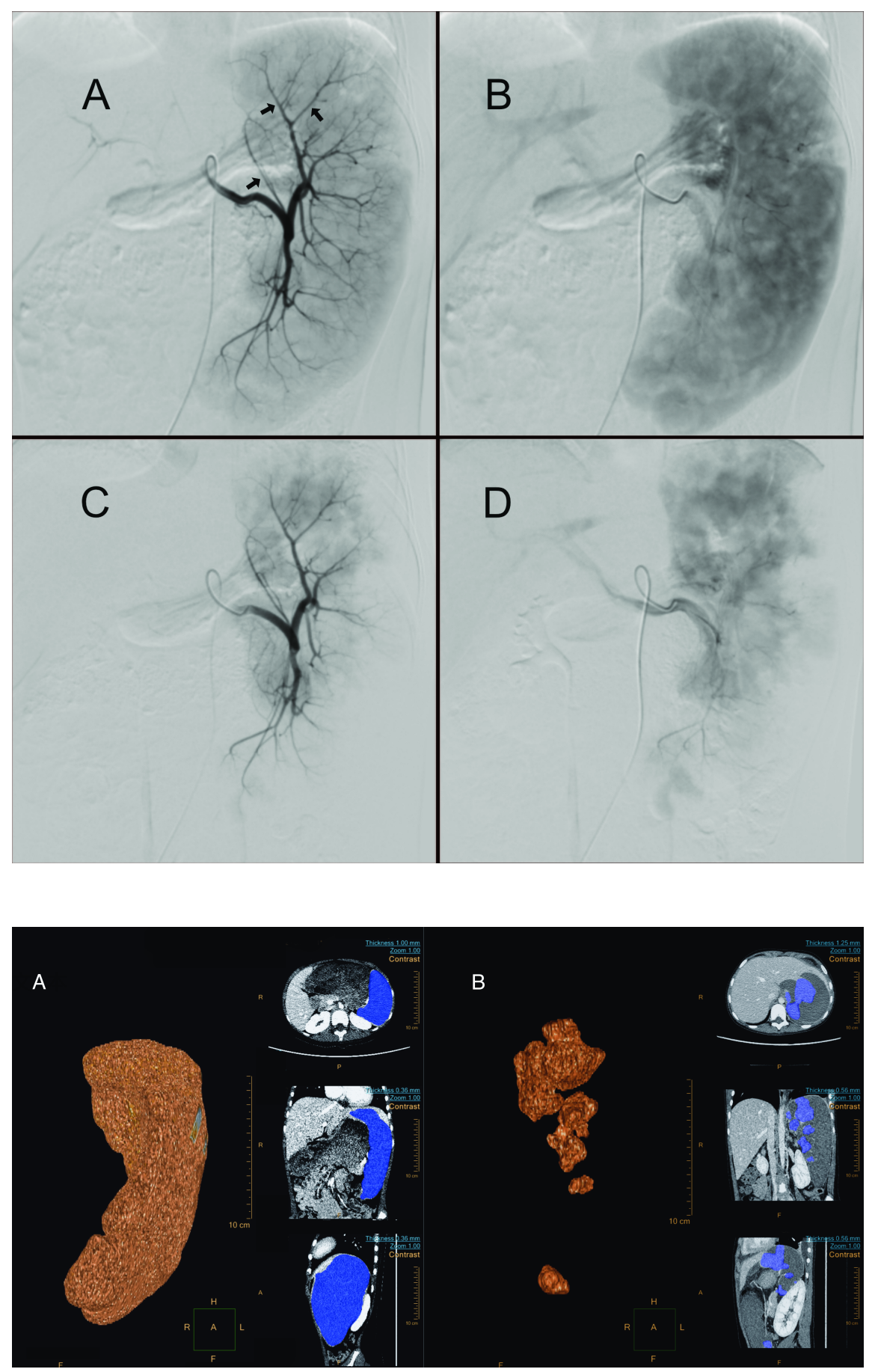